\title{
THE STRUCTURE OF (EXACTLY) 2-TO-1 MAPS ON METRIC COMPACTA
}

\author{
JO HEATH
}

(Communicated by Dennis Burke)

\begin{abstract}
It is shown that the domain of a 2-to-1 continuous map $f$ contains two disjoint open sets $V$ and $V^{\wedge}$ such that $f(V)=f\left(V^{\wedge}\right)$ and $f \uparrow V$ is a homeomorphism from $V$ onto a dense open subset of the image of $f$. The restriction of $f$ to $V \cup V^{-}$is the first "fold", and $f$ is shown to be the union of a finite or transfinite sequence of similar folds.
\end{abstract}

\section{INTRODUCTION}

A function is $k$-to- 1 if each point in the image has exactly $k$ points in its preimage. In the study of $k$-to-1 functions, the case $k=2$ is frequently an anomaly. For instance, while there are continuous $k$-to-1 functions defined on the arc $[0,1]$ for each $k>2$, infinitely many discontinuities are required for $k=2$. (See [1], [2], and [3].) More generally, if $G$ is a graph with Euler number $m$, and $H$ is a graph with Euler number $t$, then there is a finitely discontinuous $k$-to-1 function from $G$ onto $H$ iff $m \leq k t$ except for the case $k=2$ where the much stricter $m=k t$ is required. (See [4].) Another example is the fact that while $k$-to- 1 (continuous) maps have been constructed onto treelike continua for every $k>2$, it is not known if any treelike continum can be the image of a 2-to-1 map, (see [5]), nor is it known if the pseudoarc can be the domain of a 2-to-1 map. These and other examples support the notion that the structure of 2-to-1 maps is more demanding than the structure of $k$ to-1 maps for $k>2$. In this paper we study the structure of 2-to-1 maps by showing how the domain can be partitioned into manageable sets on which the function is exceptionally well behaved.

\section{Definitions AND THE INTERIOR LEMMA}

Definition. For each point $p$ in the domain of a 2-to-1 map $f$, let $p^{-}$, called the twin of $p$, denote the other point of the domain of $f$ such that

Received by the editors May 19, 1989; some parts of this paper were presented at the Spring Topology Conference in Knoxville, Tennessee, March 1989.

1980 Mathematics Subject Classification (1985 Revision). Primary 54C10, 26A15, 26 A03.

Key words and phrases. $k$-to-1 function, 2-to-1 map, $k$-to-1 map, 2-10-1 function. 
$f\left(p^{\wedge}\right)=f(p)$. Analogously, define $D^{\wedge}=\left\{x^{\wedge}: x\right.$ is in $\left.D\right\}$, for any subset $D$ of the domain of $f$.

Definition. The point $p$ in the domain $A$ of a 2-to-1 map is a hinge point if there is a sequence of points in $A$ converging to $p$ whose sequence of twins also converges to $p$.

Definition. [6, p. 12] A function $t: A \rightarrow A$ is semicontinuous if for each point $x$ and any sequence of points converging to $x$, the sequence of images under $t$ does not converge to any point other than $t(x)$ or $x$.

Fact 0. [6, p. 12] If $f: A \rightarrow B$ is 2-to-1 and continuous, then the twin function on $A$ that takes each $x$ to $x^{\wedge}$ is semicontinuous. Note that if $A$ is compact then the points of discontinuity of the twin function are exactly the hinge points.

Lemma 1 (Interior Lemma). Suppose $f: A \rightarrow B$ is continuous and 2-to-1 and $A^{\prime}$ is a compact subset of $A$ with $f\left(A^{\prime}\right)=B$. Then $A^{\prime}$ must have interior. Furthermore if $A^{\prime}$ is minimal with respect to the property of being a compact subset of $A$ that maps onto $B$, then $A^{\prime}$ is the closure of its interior.

Proof. Suppose $A^{\prime}$ has no interior and let $S$ denote the subset of $A^{\prime}$ of special hinge points that are sequential limit points of two twin sequences, one of which is in $A \backslash A^{\prime}$. The other sequence must be in $A^{\prime}$ since $f\left(A^{\prime}\right)=B . S$ is closed and Fact 1 (isolated because it is used often) implies that $S$ is nonempty.

Fact 1. If $p$ is not in $A^{\prime}$ and $p^{-}$is not in the interior of $A^{\prime}$, then $p^{-}$belongs to $S$, i.e., $p^{\wedge}$ is a hinge point with one sequence of points from outside of $A^{\prime}$ converging to $p^{\wedge}$ and its twin sequence from $A^{\prime}$ also converging to $p^{\wedge}$.

Proof of Fact 1. Let $p$ be as described. Since $p^{-}$is not in the interior of $A^{\prime}$ (but must be in $A^{\prime}$ ), there is a sequence $\{a(i)\}$ of points outside of $A^{\prime}$ converging to $p^{\curlywedge}$. The twin sequence $\left\{a(i)^{\wedge}\right\}$ in the compact set $A^{\prime}$ must converge to $p^{-}$since each convergent subsequence converges to either $p$ or $p^{\wedge}$ by the semicontinuity of the twin function. Hence $p^{\wedge}$ is in $S$.

Now divide $S$ into pieces: $M(j)=\left\{x\right.$ in $\left.S: d\left(x, x^{\wedge}\right) \geq 1 / j\right\}$. These pieces are closed so, by Baire's theorem, one of them has interior (with respect to $S$ ). Let $x$ be an interior point then of, say, $M(i)$. By the definition of $S$ there are twin sequences converging to $x$, one from outside $A^{\prime}$, one from inside $A^{\prime}$. Thus there is a point $z$ outside $A^{\prime}$ near $x$ that is within $1 / i$ of its twin $z^{-}$ in $A^{\prime}$. But we know from Fact 1 that if $z$ is in $A \backslash A^{\prime}$, then its twin is in $S$. This contradicts the definition of $M(i)$. Hence $A^{\prime}$ has interior.

Now, suppose the set $C=A^{\prime} \backslash \mathrm{Cl}\left(\operatorname{Int}\left(A^{\prime}\right)\right)$ exists, where $A^{\prime}$ is minimal with respect to being a compact subset of $A$ that maps onto $B$. Then $C$ satisfies the Baire property and can be broken down into the same sets $M(j)$ as before, and one of them, say $M(i)$, has interior in $C$. Let $V$ be an open set (in $C$ and hence in $A^{\prime}$ ) in $M(i)$ of diameter less than $1 / i$. Then $V$ and $V^{\wedge}$ do not intersect. If $V^{\wedge}$ is in $A^{\prime}$, then $A^{\prime} \backslash V$ is a closed proper subset of $A^{\prime}$ that maps onto $B$, a contradiction, so some point $q$ of $V$ has $q^{\wedge}$ not in $A^{\prime}$. As 
before, from Fact 1, since $q$ is not in the interior of $A^{\prime}$, we know $q$ must be in $S$ and the same contradiction occurs.

Corollary 1. If $f$ is continuous and 2-to-1 and maps the indecomposable continuum $A$ onto a continuum $Y$, then no proper subcontinuum of $A$ maps by $f$ onto $Y$.

Proof. If $A$ is an indecomposable continuum, then no proper subcontinuum of $A$ has interior.

\section{THE FOLDING SEQUENCE OF A 2-TO-1 MAP}

The idea of a fold can be seen in this simple example: The unit circle $S$ in the plane can be mapped 2-to-1 onto itself as a composition of $S$ projected onto $[-1,1]$ followed by gluing the points $(-1,0)$ and $(1,0)$ together. The 2-to1 composition is a homeomorphism on the open set $\mathrm{V} 1$, the points of $S$ with positive second coordinate, and on the disjoint open twin set $\mathrm{V}^{\uparrow}$, the points of $S$ with negative second coordinate, and each maps to the same dense open set in the image. We will show that all 2-to-1 maps on metric compacta behave this way. (See Corollary 2 below.) The construction of V1 is described in Theorem 1. Obviously this phenomenon dictates a certain amount of similarity between the domain and image of a 2-to-1 map; see Corollary 3. Since V1 and V1 map to the same set, the function restricted to the first residue $A \backslash\left(\mathrm{V} 1 \cup \mathrm{V} \mathrm{1}^{\wedge}\right)$ is still 2-to-1, and since $\mathrm{V} 1$ and $\mathrm{V}_{1}{ }^{-}$are open, the residue is compact. Hence the process can be repeated by constructing disjoint twin sets $\mathrm{V} 2$ and $\mathrm{V} 2{ }^{-}$open in the first residue. In the circle $S, \mathrm{~V} 2$ and V2 ${ }^{-}$will be just $\{(-1,0)\}$ and $\{(1,0)\}$, but in more complex situations, there may be a transfinite sequence of such $V^{\prime} s$ before $A$ is exhausted. (Example 3 describes a Peano continuum whose every $V$ sequence has ordinal at least $w_{0}$.) In general, we define the residue $A(\beta+1)=A \beta \backslash\left(V \beta \cup(V \beta)^{\wedge}\right)$ and $A \beta$, for limit $\beta$, is defined to be the intersection of the previous $A i^{\prime} s$ (so limit $A \beta^{\prime} s$ are always nonempty since the $A i^{\prime} s$ are compact). Since each $V \beta$ is never empty (shown in Theorem 1) if the residue isn't empty, something is glued at each step so the folding sequence eventually exhausts $A$, i.e., some first residue $A(\alpha+1)$ is empty. The residue sequence for $f$ and $A$ is $A 1, A 2, \ldots, A \beta, \ldots, A \alpha$ and the folding sequence of $f$ and $A$ is $V 1, V 2, \ldots, V \alpha$. Note that the last nonempty residue $A \alpha=V \alpha \cup(V \alpha)^{\wedge}$. This sequence of $V^{\prime} s$ as a decomposition of $A$ is a natural set-up for transfinite induction. Some of the important properties of the $V$ sequence are outlined in Theorem 2.

Theorem 1. Suppose $f: A \rightarrow B$ is continuous and 2-to-1, $A$ is compact, $A^{\prime}$ is a set minimal with respect to the property of being a closed subset of $A$ that maps by $f$ onto $B$, and suppose $V$ is the set of points in the interior of $A^{\prime}$ whose twins do not belong to $A^{\prime}$. Then $V$ is a nonempty open set such that $V^{-}$is open and disjoint from $V$, and if $V$ is properly contained in the open set $W$, then either $W^{-}$is not open or $W^{\wedge}$ intersects $W$. Furthermore, $V$ is dense in $A^{\prime}$. 
Proof. Let $f, A, B, A^{\prime}$, and $V$ satisfy the hypothesis.

Fact 2. If $x$ is in the interior of $A^{\prime}$ and $x^{-}$is in $A^{\prime}$, then $x^{-}$is a hinge point. Proof of Fact 2. Let $U$ and $W$ be disjoint open sets containing $x$ and $x^{-}$ respectively such that $U$ is in the interior of $A^{\prime}$. If $x^{-}$is not a hinge point there is, by the semicontinuity of the twin function, a smaller open set $W^{\prime}$ containing $x^{\wedge}$ such that $\left(W^{\prime}\right)^{-}$lies in $U$. But this means that $A^{\prime} \backslash W^{\prime}$ is a closed proper subset of $A^{\prime}$ that also maps onto $B$, contradicting the minimality of $A^{\prime}$. Hence $x^{\uparrow}$ is a hinge point.

Fact 3. The interior of $A^{\prime}$ has no hinge points.

Proof of Fact 3. The set of hinge points of the interior of $A^{\prime}$, if nonempty, is closed in the interior of $A^{\prime}$ and so satisfies the Baire property. As in the proof of Lemma 1 , let $M(j)=\left\{x: d\left(x, x^{\widehat{\gamma}}\right) \geq 1 / j\right\}$ for each positive integer $j$; then there is a point $x$ in the interior of some $M(i)$. Since $x$ is a hinge point, there are pairs of twins arbitrarily close to $x$ most of which cannot be in $M(i)$ and so cannot be hinge points themselves. Thus there is in the interior of $A^{\prime}$ a twin pair of points neither of which is a hinge point, contrary to Fact 2.

Claim 1. $V$ is dense in $A^{\prime}$. The interior $I$ of $A^{\prime}$ is dense in $A^{\prime}$ by Lemma 1 ; to show that $V$ is dense in $I$, let $W$ denote any open set in $I$. If each $x$ in $W$ has $x^{-}$in $A^{\prime}$, then since $x^{-}$is a hinge point (Fact 2), it is not in $I$ (Fact 3), and so $W$ and $W^{\wedge}$ are disjoint and $A^{\prime} \backslash W$ maps onto the image $B$, contrary to the minimality of $A^{\prime}$. Hence some point $x$ of $W$ has $x^{-}$not in $A^{\prime}$, i.e., $x$ is in $V$.

Claim 2. $V$ is open. If the sequence $\{x(i)\}$ converges to $p$ in $V$, then their twins converge to $p^{\widehat{\lambda}}$ in $A \backslash A^{\prime}$, since $p$ is not a hinge point. Then, since most of the $x(i)$ must be in the interior of $A^{\prime}$ since $p$ is, all but finitely many of the $x(i)$ are in $V$. Hence $V$ is open.

Claim 3. $V^{-}$is open (and obviously disjoint from $V$ ). Let $\{x(i)\}$ be a sequence of points in $A \backslash A^{\prime}$ converging to the point $q$ of $V^{\frown}$. Since $f\left(A^{\prime}\right)=B$, each $x(i)^{\wedge}$ is in $A^{\prime}$ and hence $\left\{x(i)^{\wedge}\right\}$ converges to $q^{-}$in $V$. Since $V$ is open, most $x(i)^{\wedge}$ are in $V$ and for these $x(i)^{\wedge}, x(i)$ is in $V^{\wedge}$; hence $V^{\wedge}$ is open.

Now suppose that $W$ is a larger open set containing $V$ with the property that $W^{\wedge}$ is open and misses $W$. If $W$ is not in the interior of $A^{\prime}$, then $W$ contains a point $p$ not in $A^{\prime}$, since $W$ is open. If $p^{\wedge}$ is in the interior of $A^{\prime}$, it belongs to $V$ and hence to $W$ so that $W$ intersects $W^{\wedge}$; so $p^{\wedge}$ must be in the boundary of $A^{\prime}$ since it must be in $A^{\prime}$. But, by Fact $1, p^{-}$is a hinge point, so $W$ intersects $W^{\wedge}$. Thus $W$ is contained in the interior of $A^{\prime}$. Since $W \neq V, W$ has a point $q$ whose twin is in $A^{\prime}$. But this means that $A^{\prime}$ intersects $W^{\wedge}$, and $A^{\prime} \backslash W^{\wedge}$ is a closed proper subset of $A^{\prime}$ that maps onto $B$ (a contradiction). Therefore there is no such larger $W$. 
Corollary 2. If the 2-to-1 function $f$ maps the compactum $A$ onto $B$, then there are disjoint open twin sets $V$ and $V^{\wedge}$ in $A$ such that $f$ restricted to either of them is a homeomorphism and $f(V)$ is open and dense in $B$.

Proof. Let $V$ and $A^{\prime}$ be as described in Theorem 1. Then $f(V)$ is dense in $B$ since $A^{\prime}$ maps onto $B$ and $V$ is dense in $A^{\prime}$. Since $V$ and $V^{-}$do not intersect, $f$ is 1-to-1 on each of them; and it is straightforward to show, since $V \cup V^{\wedge}$ is the complete inverse of $f(V)$, that $f(V)$ is open in $B$, and that $f$ is a homeomorphism when restricted to either $V$ or $V^{\wedge}$.

Corollary 3. Given a hereditarily decomposable continuum and a hereditarily indecomposable continuum, neither can be mapped 2-to-1 onto the other.

Corollary 4. If $f: A \rightarrow B$ is 2-to-1 and continuous, $A$ is compact, and $A^{\prime}$ is a set minimal with respect to being a closed subset of $A$ such that $f\left(A^{\prime}\right)=B$, then $f$ is 1-to-1 on each of the sets $\operatorname{Int}\left(A^{\prime}\right)$ and $A \backslash A^{\prime}$; hence the set of points at which $f$ is a local homeomorphism contains the dense open set $\operatorname{Int}\left(A^{\prime}\right) \cup\left(A \backslash A^{\prime}\right)$.

Note. The set of points at which $f$ is a local homeomorphism was shown by Mioduszewski [6] and Roberts [7] to be open and dense.

Proof. The function $f$ is 1-to- 1 on $A \backslash A^{\prime}$ because $A^{\prime}$ maps onto $B$, and Facts 2 and 3 imply $f$ is 1 -to-1 on $\operatorname{Int}\left(A^{\prime}\right)$.

The following theorem is a gathering of some of the properties of the folding sequence that might be useful for transfinite induction:

Theorem 2. Suppose $f: A \rightarrow B$ is continuous and 2-to-1, and $A$ is compact. Then there is an increasing sequence $W 1, W 2, \ldots, W \beta, \ldots, W \alpha$ of open sets in $A$ such that (1) $W \alpha=A$, (2) each $W \beta=(W \beta)^{\wedge}$, (3) each $W \beta \backslash$ $\bigcup\{W i ; i<\beta\}$ is the union of two nonempty separated twin sets $V \beta$ and $(V \beta)^{\wedge}$, and (4) if $T=f^{-1}(Z)$ for some compactum $Z$ in the image $B$, and $\beta$ is the least ordinal such that $T$ is contained in $W \beta$, then $T$ intersects $V \beta \cup(V \beta)^{\wedge}$, and the twin function (that takes each $x$ to $x^{\wedge}$ ) is a homeomorphism from $T \cap V \beta$ onto $T \cap(V \beta)^{\wedge}$.

Proof. For each $j$, let $W j=U\left\{V i \cup(V i)^{\wedge}: i \leq j\right\}$. Then properties (1) through (3) follow immediately from the construction of a folding sequence. For (4) suppose $\beta$ is a limit ordinal. Since $T$ is not in $W \alpha$ for any $\alpha<\beta, T$ intersects each (compact) residue $A \alpha$ and hence their intersection $A \beta$. These intersection points must be in $V \beta \cup(V \beta)^{\wedge}$. The sets $T \cap(V \beta)$ and $T \cap(V \beta)^{\wedge}$ are disjoint because $V \beta$ and $(V \beta)^{\wedge}$ are, and they are compact because limit poiints of $V \beta \cup(V \beta)^{\wedge}$ not in $V \beta \cup(V \beta)^{\wedge}$ are in $V \mu$ for some $\mu>\beta$, which is not possible if $T$ is contained in $W \beta$. That the twin function is a homeomorphism follows from the semicontinuity of the twin function (Fact 0 ), since then the twin function is continuous, 1-to-1 and onto, and $T \cap(V \beta)$ is compact. 


\section{EXAMPLES}

An example is given (Example 1) to show that although it is true that for each $x$ in $A$ either $x$ or $x^{\wedge}$ is in the closure of $V 1$, nevertheless $V 1 \cup V 1^{-}$ need not be dense in $A$. The interior of $A^{\prime}$ is dense in $A^{\prime}$, so the union $\operatorname{Int}\left(A^{\prime}\right) \cup\left(A \backslash A^{\prime}\right)$ is not an insignificant portion of $L$ (the set of points at which $f$ is a local homeomorphism) but an example is given (Example 2) where this union is a proper subset of $L$ and this same example demonstrates that the first residue, $A \backslash\left(V 1 \cup V 1^{\wedge}\right)$ need not be unique. Common to all three examples, $S$ denotes a circle, and $\mathrm{f} 1$ is a 2-to-1 map from $S$ onto $S$.

Example 1. There is a Peano continuum $A$ in $E^{3}$ and a 2-to-1 map $f$ on $A$ such that a maximal $V$ as described in Theorem 1 does not have $V \cup V^{\wedge}$ dense in $A$.

Construction. Let $A$ be the union of the following three sets:

$$
\begin{aligned}
& S=\left\{(x, y, z):(x-1)^{2}+y^{2}=1 \text { and } z=0\right\}, \\
& T=\left\{(x, y, z):(x-2)^{2}+y^{2}=4 \text { and } z=0\right\}, \text { and } \\
& R=\left\{(x, y, z):(x+2)^{2}+y^{2}=4 \text { and }-1 \leq z \leq 1\right\},
\end{aligned}
$$

and let $f$ be $\mathrm{f} 1$ on $S$, glue each point $(x, y, z)$ of $R$ with $z \neq 0$ to $(x, y,-z)$, and glue each point $(x, y, 0)$ of $R$ to the point $(-x, y, 0)$ of $T$. A suitable $V$ could be the set of points in $R$ with positive $z$ coordinate plus some of the points of $S$, and $V^{\wedge}$ would be the points of $R$ with negative $z$ coordinate plus some other points of $S$, but most points of $T$ are not in the closure of $V \cup V^{\wedge}$.

Example 2. There is a Peano continuum $A$ in $E^{3}$ and a 2-to-1 map $f$ defined on $A$ such that (1) if $A^{\prime}$ is any subset of $A$ that is minimal with respect to being a compactum that maps onto the image of $A$, then $\operatorname{Int}\left(A^{\prime}\right) \cup\left(A \backslash A^{\prime}\right)$ is a proper subset of the set $L$ of points at which $f$ is a local homeomorphism (nonhinge points), and (2) the first residue $A 2=A \backslash\left(V 1 \cup V 1^{\wedge}\right.$ ) is not unique.

Contruction. Let $A$ be the union of the following two sets:

$$
S=\left\{(x, y, z):(z-1)^{2}+x^{2}=1 \text { and } y=0\right\} \text {, and }
$$

$T=\left\{(x, y, z): x^{2}+y^{2} \leq 1\right.$ and $\left.z=0\right\}$, and let $f$ be f1 on $S$ and glue each point $(x, y, 0)$ in $T$ to $(-x,-y, 0)$ in $T$. All of the hinge points of $f$ are in $S$ and since $\operatorname{Int}\left(A^{\prime}\right)$ and $A \backslash A^{\prime}$ are disjoint nonempty open sets for any $A^{\prime}$, these two sets cannot divide up the connected set $T \backslash\{(0,0,0)\}$ and yet $L$ contains $T \backslash\{(0,0,0)\}$. Note too that $T \backslash\{(0,0,0)\}$ is neither (1) in $\operatorname{Int}\left(A^{\prime}\right)$ since $A^{\prime}$ is minimal nor (2) in $A \backslash A^{\prime}$ since $A^{\prime}$ maps onto the image. Now choose as a minimal compactum a set $A^{\prime}$ such that $A^{\prime} \cap T=$ $\left\{(x, y, z): x^{2}+y^{2} \leq 1, y \leq 0\right.$, and $\left.z=0\right\}$. The residue resulting from this $A^{\prime}$ would intersect $T$ in the diameter $\{(x, y, z):-1 \leq x \leq 1, y=0$ and $z=0\}$. Since $f$ is symmetric about the origin, any diameter could likewise be the $T$ part of some residue. Thus $A 2$ is not unique. 
Example 3. An example of a continuum $A$ in the plane and a 2-to-1 map $f$ defined on $A$ such that every folding sequence for $A$ and $f$ has ordinal at least $w_{0}$.

Contruction. Let $S$ be the unit circle in the plane and define $f$ to be $f 1$ on $S$. We will add infinitely many dendrites of sufficient complexity that the resulting continuum has hinge points remaining in every residue with integer subscript. We use the fact that no $V \alpha$ or $V \alpha^{\widehat{\gamma}}$ has any hinge points for the $\alpha$ th residue.

Suppose $E$ is an arc, $p$ is its midpoint, and $e$ is a positive number. We will very temporarily use the term "amplify $E$ with $e$ control" to mean (1) choose a sequence of points $t(i)$ on $E$ converging to $p$ and at each $t(i)$ attach the midpoint of an arc $a(i)$ of diameter less that $e / i$, and (2) extend $f$ already defined on $E$ to each $a(i)$ by gluing the two halves of $a(i)$ to each other pivoting at the midpoint $t(i)$ of $a(i)$. Now, let $E$ be a subarc of $S$ and $p$ its midpoint. The first extension is to amplify $E$ with control 1. Let $\{t(i)\}$ denote the selected points on $E$ converging to $p$ used for this amplification. The second extension is to amplify each arc added at extension one with control $1 / 2$ except for the first arc attached at $t(1)$. The $n$th extension is to amplify each arc added at extension $n-1$ with control $1 /\left(2^{n}\right)$ except for the arcs that connect to $S$ at any of the first $n$ points $t(1), \ldots, t(n)$. Let $A$ denote $S$ plus all of these extensions.

Each midpoint of each added arc is a hinge point for $A$ and so cannot be in $V 1 \cup V 1^{\wedge}$, no matter which $V 1$ is chosen. The first midpoint $t(1)$ is not however a hinge point for the first residue $A \backslash\left(V 1 \cup V 1^{\wedge}\right)$, but all hinge points that are limits of hinge points will remain in the second residue. The complexity of the hinge points as constructed guarantees that the $n$th residue is nonempty for each positive integer $n$ so that the ordinal of the folding sequence is at least $w_{0}$ no matter which sequence is chosen.

\section{REFERENCES}

1. O. G. Harrold, The non-existence of a certain type of continuous transformation, Duke Math. J. 5 (1939), 789-793.

2. Jo Heath, Every exactly 2-to-1 function on the reals has an infinite number of discontinuities, Proc. Amer. Math. Soc. 98 (1986), 369-373.

3. __ K-to-1 functions on arcs for K even, Proc. Amer. Math. Soc. 101 (1987), 387-391.

4. $\ldots$ - K-to-1 functions between graphs with finitely many discontinuities, Proc. Amer. Math. Soc. 103 (1988), 661-666.

5. __, Tree-like continua and exactly k-to-1 functions, Proc. Amer. Math. Soc. 105 (1989),

6. J. Mioduszewski, On two-to-one continuous functions, Dissertationes Math. (Rozprawy Mat.) 24 (1961), 42.

7. J. H. Roberts, Two-to-one transformations, Duke Math. J. 6 (1940), 256-262.

Foundations, Topology, and Analysis, Mathematics Department, Auburn University, Auburn, Alabama 36849-5310. 